Original Article

\title{
Evaluation of Oxidative Stress Parameters and Urinary Deoxypyridinoline Levels in Geriatric Patients with Osteoporosis
}

\author{
Mehmet Demir ${ }^{1)}$, Turgay Ulas ${ }^{1{ }^{*}}$, Ahmet Tutoglu ${ }^{2)}$, Ahmet Boyaci ${ }^{2)}$, Emel Yigit Karakas ${ }^{1)}$, \\ Hatice Sezen ${ }^{3)}$, Murat Ustunel ${ }^{3)}$, Hasan Bilinc ${ }^{3)}$, Mehmet Gencer ${ }^{4)}$, \\ HAKAN BuyUKhatipogLu' ${ }^{1)}$ \\ 1) Department of Internal Medicine, Faculty of Medicine, Harran University: Yenisehir Campus, \\ 63000, Sanliurfa, Turkey \\ 2) Department of Physical Therapy and Rehabilitation, Harran University School of Medicine, Turkey \\ 3) Department of Biochemistry, Harran University School of Medicine, Turkey \\ 4) Department of Pulmonary Diseases, Harran University School of Medicine, Turkey
}

\begin{abstract}
Purpose] To evaluate the oxidative stress parameters and urinary deoxypyridinoline levels in geriatric patients with osteoporosis. [Subjects and Methods] Eighty geriatric patients aged over 65 years were recruited. Patients were divided into two groups: Group $1(n=40)$ consisted of patients with osteoporosis, and Group $2(n=40)$ consisted of patients without osteoporosis. Bone mineral density measurements were performed for all patients using DEXA. Oxidative stress parameters were analyzed in blood samples, and deoxypyridinoline levels were analyzed in 24-hour urinary samples. [Results] Compared to Group 2, the total antioxidant status and oxidative stress index levels of Group 1 were not significantly different; however, total oxidant status and 24-hour urinary deoxypyridinoline levels were significantly higher. Pearson correlation coefficients indicated that OSI and urinary deoxypyridinoline levels were not correlated with any biochemical parameters. ROC-curve analysis revealed that urinary deoxypyridinoline levels over $30.80 \mathrm{mg} / \mathrm{ml}$ predicted osteoporosis with $67 \%$ sensitivity and $68 \%$ specificity (area under the curve $=0.734 ; \% 95$ CI: $0.624-0.844$ ). [Conclusion] Our results indicate that oxidative stress would play a role in the pathogenesis of osteoporosis, and that urinary deoxypyridinoline levels may be a useful screening test for osteoporosis.

Key words: Osteoporosis, Oxidative stress index, Deoxypyridinoline
\end{abstract}

(This article was submitted Jan. 27, 2014, and was accepted Mar. 9, 2014)

\section{INTRODUCTION}

Osteoporosis (OP) is defined as a skeletal disease characterized by bone fragility due to low bone mass and deterioration of bone microstructure ${ }^{1)}$. In the diagnosis of osteoporosis, bone mineral density measurement methods and biochemical examination play important roles as well as detailed history and physical examination of the patient ${ }^{2}$. Imaging methods have an important role in diagnosis and monitoring of osteoporosis and are used for general purposes such as determination the degree of osteoporosis and fracture risk, monitoring the rate of bone loss and monitoring the effectiveness of treatment. Dual energy X-ray absorptiometry (DEXA) is the most preferred method of measuring bone mineral density. Non-invasive techniques for measuring the bone turnover provide significant advan-

*Corresponding author. Turgay Ulas (E-mail: turgayulas@ yahoo.com)

(C2014 The Society of Physical Therapy Science. Published by IPEC Inc. This is an open-access article distributed under the terms of the Creative Commons Attribution Non-Commercial No Derivatives (by-ncnd) License $<$ http://creativecommons.org/licenses/by-nc-nd/3.0/> . tages in the follow-up diagnosis and treatment of osteoporosis. For example urine and serum markers can be used to measure enzymes secreted by osteoblasts or osteoclasts, or non-enzymatic peptides during formation and resorption of bone. Biochemical markers (osteocalcin, peptides containing pyridinoline, total and bone alkaline phosphatase etc.) can be used for screening or diagnostic purposes in the evaluation of metabolic bone disease, and they are also used to evaluate the utility of therapeutic regimens ${ }^{3-5}$ ).

The bone is a complex tissue which is composed of various cell types such as osteoclasts and osteoblasts, and these cells are the primary actors of an ongoing renewal and repair cascade called bone reconstruction (or remodeling). In this cascade, there is a balance between the activities of both types of cells and the balance is coordinated carefully by various hormones or cytokines ${ }^{6-8)}$. Oxidative stress denotes a serious imbalance between free radical formation and antioxidant defense mechanisms, and ultimately leads to tissue damage due to this imbalance. Earlier studies have investigated possible relationships between bone mineral density and oxidative stress but it is still not clear whether or not oxidative stress has a significant role in the development of osteoporosis since conflicting results have been 
reported $^{9-11)}$

Although there are numerous studies in the literature that have investigated the levels of oxidative stress and urinary deoxypyridinoline in osteoporosis patients, to the best of our knowledge, no study has been conducted of geriatric patients. We, therefore, assessed the levels of deoxypyridinoline in urine and oxidative stress and compared them with bone density measurements in geriatric osteoporosis patients.

\section{SUBJECTS AND METHODS}

Patients admitted to the Harran University School of Medicine, between August 2011 and January 2012, who were over the age of 65 were included in this cross-sectional study. Written consent to participation in this study was received from the patients and the ethics committee of our hospital granted approval, conforming to the principles of the 2nd Declaration of Helsinki.

Eighty consecutive geriatric patients who were not subject to the exclusion criteria were divided into two groups. Group $1(\mathrm{n}=40)$ comprised patients with osteoporosis and Group $2(n=40)$ comprised without osteoporosis with similar demographic characteristics. The exclusion criteria were patients with endocrine diseases thought to cause osteoporosis (thyroid, diabetes mellitus, prolactinoma, Cushing syndrome, hyperparathyroidism); those receiving osteoporosis treatment, steroids, thiazide diuretics, anticoagulants, LH-RH agonist, anticonvulsants, pioglitazone, heparin or methotrexate; those receiving antacids therapy containing aluminum; those with kidney, liver disease or celiac disease; those with a history of gastrectomy operation; those who had malabsorbtion, or total parenteral nutrition; those who were immobile; those who were smoking and using alcohol; and those who had a history of early menopause.

The bone density of the subjects was measured using DEXA and T scores in the L2-L4 vertebrae and femoral neck under -2.5 were considered as osteoporotic. Oxidative stress parameters of venous blood samples and deoxypyridinoline levels in 24-hour urine samples were also measured.

A Hologic QDR 4500 A DEXA (Hologic INC 02154USA) device was used for BMD measurements of patients and BMD of the Ll-L4 vertebrae, femoral neck and total femur density was expressed as $\mathrm{g} / \mathrm{cm}^{2}$. L1-L4 averages were used as the vertebral BMD. The body mass index (BMI = Body Mass Index) was calculated by dividing body weight by the square of height.

Five mililiter blood samples were taken from forearm venous blood vessel of each subject and placed in Eppendorf tubes. The tubes were centrifuged at $1,500 \mathrm{rpm}$ for $10 \mathrm{~min}$ utes to obtain serum samples for the measurements of total oxidant status (TOS) and total antioxidant status (TAS). All serum samples were stored at $-80^{\circ} \mathrm{C}$ after labeling in the biochemistry laboratory until the day of analysis.

Serum TOS was measured using a novel automated method developed by Erel ${ }^{12}$. Oxidants present in the sample oxidize the ferrous ion-o-dianisidine complex to ferric ion. The oxidation reaction is enhanced by glycerol molecules, which are abundantly present in the reaction medium. The ferric ion generates a colored complex with Xylenol Orange in an acidic medium. The color intensity, which can be measured spectrophotometrically (V-530; Jasco ${ }^{\circledR}$, Tokyo, Japan), is related to the quantity of oxidant molecules present in the sample. The assay is calibrated with hydrogen peroxide, and the results are expressed in terms of micromolar hydrogen peroxide equivalents per liter $\left(\mu \mathrm{mol} \mathrm{H}_{2} \mathrm{O}_{2}\right.$ equiv./l).

Serum TAS was measured using a novel automated method developed by Erel $^{13)}$. In this method, hydroxyl radical, the most potent biological radical, is produced. In the assay, the ferrous ion solution in reagent 1 is mixed with the hydrogen peroxide present in reagent 2 . Sequentially produced radicals, such as the hydroxyl radical-produced brown-colored dianisidinyl radical cation, are also potent radicals. This method allows the measurement of the sample's antioxidative effect against potent free radical reactions that are initiated by the hydroxyl radical. The assay has excellent precision values of more than $97 \%$. The results are expressed as mmol Trolox equiv./1.

The OSI was defined as the ratio of TOS to TAS level. A standardized value does not exist for OSI levels, which were used only for comparisons. For the calculation, TAS units were changed to $\mathrm{mmol} / \mathrm{l}$, and the OSI value was calculated according to the following formula: OSI (arbitrary unit) = TOS ( $\mu$ mol $\mathrm{H}_{2} \mathrm{O}_{2}$ equiv./l)/TAS (mmol Trolox equiv./l) ${ }^{14,15)}$.

Deoxyprydoline in 24-hour urine samples was assayed by the chemiluminescence method in Immulite 2000 hormones (Siemens).

Serum urea, creatinine, fasting blood glucose, aspartate aminotransferase, alanine aminotransferase, albumin, alkaline phosphatase, sodium, potassium, calcium, phosphor, magnesium, C-reactive protein, free T3, free T4, adrenocorticotropic hormone, cortisol, parathyroid hormone, glycated haemoglobin, triglycerides, total cholesterol, and high-density and low-density lipoprotein cholesterol levels were analyzed using a Cobas Integra 800 auto analyzer (Roche) and commercial measurement kits (Roche).

SPSS 18.0 was used for all statistical analyses (Illniosis, Chicago, USA). The one Sample Kolmogorov-Smirnov test was used to verify the normality of data distributions. Results are expressed as mean $\pm \mathrm{SD}$. The $\chi^{2}$ test was used for categorical variables. The independent sample $t$ test was used to analyze parametric numerical data, and the MannWhitney $\mathrm{U}$ test was used to analyze non-parametric data. Pearson correlation coefficients were used to determine correlations between oxidative stress and deoxypyridinoline levels with the other parameters of the osteoporosis patients. Binary logistic regression analysis was performed to find independent predictors of osteoporosis. Receiver operating curve (ROC) analysis was performed to assess the value of deoxypyridinoline levels to detect osteoporosis. Values of $p<0.05$ were considered statistically significant for all results.

\section{RESULTS}

Patient's overall clinical, anthropometric, biochemical findings are shown in Table 1. Statistically significant 
differences were not found between the groups in terms of age or gender $(\mathrm{p}>0.05)$. Although the TAS and OSI values of Group 1 patients were not significantly different from those of Group 2 patients ( $p>0.05$ ), their TOS and urine deoxypyridinoline levels were significantly higher $(p<0.05)$. In Pearson correlation analysis, urinary deoxypyridinoline and OSI values were not associated with any examined parameter (for all $\mathrm{p}>0.05$ ).

Binary logistic regression analysis revealed that deoxypyridinoline levels were an independent factor $(\mathrm{B}=$ $0.017, \mathrm{SE}=0.07$, Wald $=5.87, \mathrm{p}=0.015)$, whereas OSI levels were not an independent factor of osteoporosis $(B=-0.241$, $\mathrm{SE}=0.19$, Wald $=1.51, \mathrm{p}=0.218$ ). In ROC-curve analysis, urine deoxypyridinoline levels above $30.80 \mathrm{mg} / \mathrm{ml}$ showed $67 \%$ sensitivity and $68 \%$ specificity in the diagnosis of osteoporosis.

\section{DISCUSSION}

This study is the first study to investigate urine deoxypyridinoline levels with oxidative stress parameters in geriatric osteoporosis patients and the main results are: (i) urinary deoxypyridinoline levels were higher in patients with osteoporosis, (ii) the oxidative stress index was similar in both groups, (iii) There was no relationship between levels of oxidative stress and urine deoxypyridinoline, and (iv) the urinary deoxypyridinoline level is a moderately accurate method for the diagnosis of geriatric osteoporosis.

Osteoporosis is a disease characterized by increased bone fragility due to decrease of bone density. It is an important public health problem because it affects the quality of patients' lives, complications require prolonged and expensive treatments, and it may even lead to death ${ }^{16,17)}$ The role of oxidative stress in patients with osteoporosis has been studied in many studies, but the results are contradictory in general.

The results of various studies suggest that oxidative stress plays an important role in the pathophysiology of osteoporosis ${ }^{9)}$. It has been argued that increased reactive oxygen species are not the cause of osteoporosis, rather they are a secondary development of a primary disorder ${ }^{18)}$. An increase in oxidative stress during the menopausal period, and decreases in antioxidants such as glutathione in erythrocytes, total thiol, $\alpha$-tocopherol and ascorbic acid have been demonstrated, and it is known that free oxygen radicals play an important role in bone resorption $\left.{ }^{10}, 11\right)$. Moreover, it is widely accepted that bone is formed by differentiation of osteoclasts ${ }^{19-23)}$.

Epidemiological data support the hypothesis that antioxidants decrease the incidence of osteoporosis ${ }^{24)}$. In postmenopausal osteoporotic women, Sontakke ${ }^{25}$ ) and Maggio $^{23)}$ found significantly lower levels of glutathione peroxidase which is a potent antioxidant enzyme in plasma. In another study, a significant decrease in TAS and a significant increase in TOS values were found in postmenopausal women ${ }^{10)}$. In a study that compared oxidative stress and antioxidant capacity with osteoporosis patients and healthy controls, a significant decrease in TAS and significant increases in TOS and OSI values were found in postmeno-
Table 1. General demographics, clinical data, and biochemical findings of the subjects of this study

\begin{tabular}{lcc}
\hline & $\begin{array}{c}\text { Group } 1 \\
(\mathrm{n}=40)\end{array}$ & $\begin{array}{c}\text { Group } 2 \\
(\mathrm{n}=40)\end{array}$ \\
\hline Age (years) & $71.77 \pm 6.47$ & $70.0 \pm 4.8$ \\
Gender (M / F) & $30 / 10$ & $28 / 12$ \\
BMI (kg/m2) & $28.6 \pm 2.8$ & $27.5 \pm 3.9$ \\
Fasting BG (mg/dL) & $105.37 \pm 14.22$ & $106.20 \pm 17.64$ \\
Creatinine (mg / dL) & $0.73 \pm 0.16$ & $0.70 \pm 0.17$ \\
Urea (mg / dl) & $33.6 \pm 8.4$ & $31.3 \pm 8.7$ \\
ALT (U/L) & $17.8 \pm 7.4$ & $20.10 \pm 7.63$ \\
AST (U/L) & $19.5 \pm 6.2$ & $21.5 \pm 7.6$ \\
Albumin (g/dL) & $3.19 \pm 0.66$ & $3.24 \pm 0.52$ \\
ALP (U/L) & $93.0 \pm 30.2$ & $85.8 \pm 30.1$ \\
Ca (mg/dL) & $9.28 \pm 0.63$ & $9.21 \pm 0.05$ \\
Phosphorus (mg/dl) & $3.46 \pm 0.57$ & $3.31 \pm 0.56$ \\
T. cholesterol (mg/dl) & $191.32 \pm 41.02$ & $179.80 \pm$ \\
Mg (mg/dL) & $1.99 \pm 0.28$ & $1.99 \pm 0.23$ \\
LDL (mg/dL) & $112 \pm 30.7$ & $103.7 \pm 40.4$ \\
HDL (mg/dL) & $46.8 \pm 10.9$ & $45.3 \pm 13.5$ \\
TG (mg/dL) & $162.27 \pm 90.4$ & $156.52 \pm 80.5$ \\
TSH (mIU/L & $1.78 \pm 1.07$ & $1.95 \pm 1.16$ \\
PTH (pg/mL) & $83.05 \pm 48.1$ & $58.2 \pm 19.2$ \\
Sedimentation mm/h & $24.02 \pm 13.8$ & $21.6 \pm 11.0$ \\
CRP (mg/dL) & $1.87 \pm 2.57$ & $1.24 \pm 0.86$ \\
Hemoglobin (g/dL) & $12,70 \pm 1.49$ & $15.8 \pm 2.01$ \\
OSI Arbitrary Units & $3.44 \pm 1.20$ & $3.01 \pm 1.24$ \\
Tos $\mu$ mol H $\mathrm{O}_{2}$ Equiv. / L & $31.8 \pm 10.0$ & $25.6 \pm 7.4 *$ \\
Tas $\mu \mathrm{molTrolox} \mathrm{Equiv.} \mathrm{/} \mathrm{L}$ & $0.94 \pm 0.13$ & $0.89 \pm 0.18$ \\
DPD (mg/ml) & $63.8 \pm 48$ & $36.3 \pm 36.2 *$ \\
\hline p $00.05 *$ & & \\
& &
\end{tabular}

$$
\mathrm{p}<0.05^{*}
$$

pausal women ${ }^{26)}$. In the study of Wolf et al. ${ }^{27)}$, no significant relationship was found between bone mineral density and serum antioxidants in postmenopausal women. In our present study, the TAS and OSI values of geriatric osteoporosis patients were similar to those of the geriatric healthy controls, though their TOS levels were higher.

Bone turnover is a cycle consisting of successive periods of bone formation, bone resorption and mineralization that provide vitality to bone tissue. Bone turnover is inhibited by aging and deterioration of the bone. Biochemical markers in serum and urine are used to estimate bone formation and resorption rates in the turnover process. These markers in patients with osteoporosis are used to determine the rate of bone loss and the effectiveness of the treatment. Bone mineral density and its microarchitecture are the main determinants of bone strength and are essential factors in the evaluation of the mechanical properties of bone. The bone turnover is the principal factor determining bone mineral density and its microarchitecture. Accordingly, it is an expected finding that biochemical markers of bone turnover are related to bone mineral density ${ }^{28,29}$.

Deoxypyridinoline is a by-product of bone turnover. In the literature, there are many studies of deoxypyridinoline 
levels in osteoporosis patients, and in general, levels have been shown to increase than in patients with osteoporosis. In a study of post-menopausal women conducted by Ohta et al., it was found that the urinary hydroxyproline/creatinine ratio, and serum levels of osteocalcin are significantly increased in post-menopausal women ${ }^{30}$. Although Minure et al. found no correlation between BMD and biochemical markers in premenopausal women, they found a negative correlation between procollagen carboxyterminal propeptide, pyridinoline and ALP values with BMD, and a positive correlation between the amount of carboxyterminal propeptide and bone loss in postmenopausal women ${ }^{31}$. Garnero et al. found the highest levels of osteocalcin, NTX, CTX and bone ALP levels in women with the lowest bone density, in a study of 653 menopausal European women conducted over more than 20 years. In the same study, bone turnover rates of elderly women with low bone density BMD were found to be $85 \%$ higher than those of normal women ${ }^{29)}$.

There are conflicting results concerning the possible relationships between bone mineral density and biochemical indicators in the literature. In the study of Senocak et al. of 35 postmenopausal osteoporotic and 15 postmenopausal healthy volunteers, no relation was found between BMD and total alkaline phosphatase, procollagen carboxy peptide, urine concentrations of calcium, and deoxpyridinoline levels ${ }^{32}$. In a study of 30 postmenopausal osteoporotic and 30 postmenopausal healthy volunteers, a negative correlation was found between BMD and the osteocalcin level ${ }^{33)}$ In our present study, urine deoxypyridinoline levels in geriatric patients were higher than in healthy controls.

To the best of our knowledge, the feasibility of deoxypyridinoline levels as a screening test for patients with osteoporosis has not been previously investigated. Deoxypyridinoline levels are cheap and easy to measure, and could be a useful marker of osteoporosis. Our present results suggest that it is an independent predictor of osteoporosis, and a moderately accurate marker of osteoporosis in geriatric patients. We found that deoxypyridinoline levels above $30.80 \mathrm{mg} / \mathrm{ml}$ have $67 \%$ sensitivity and $68 \%$ specificity in the diagnosis of geriatric osteoporosis.

There are various limitations to the interpretation of our results to consider. The small number of subjects, and the cross-sectional design are its main constraints. Also, the moderate sensitivity and specifity of deoxypyridinoline as an indicator of osteoporosis may be associated with small number of tested subjects.

In conclusion, our study results did not find evidence that oxidative stress plays a role in the pathogenesis of osteoporosis; however, deoxypyridinoline levels can be used as a screening test for this disease, and they may be useful for early diagnosis or follow-up of this disease. Randomized prospective studies need to be conducted with larger numbers of subjects to confirm our novel results.

\section{REFERENCES}

1) Tanaka R, Ozawa J, Umehara T, et al.: Does exercise intervention improve muscle strength and balance of japanese subjects with osteoporosis?: a systematic review and meta-analysis of randomized controlled trials. J Phys
Ther Sci, 2013, 25: 397-401. [CrossRef]

2) Nakamura $K$, Tsugawa N, Saito T, et al.: Vitamin D status, bone mass, and bone metabolism in home-dwelling postmenopausal Japanese women: Yokogoshi Study. Bone, 2008, 42: 271-277. [Medline] [CrossRef]

3) Kuchuk NO, van Schoor NM, Pluijm SM, et al.: Vitamin D status, parathyroid function, bone turnover, and BMD in postmenopausal women with osteoporosis: global perspective. J Bone Miner Res, 2009, 24: 693-701. [Medline] [CrossRef]

4) Delmas PD: Markers of bone formation and resorption. In: Primer on the metabolic bone diseases and disorders of mineral metabolism. New York: Lippincott Raven, 1993, pp 30-31.

5) Delmas PD: Biochemical markers of bone turnover for the clinical investigation of osteoporosis. Osteoporos Int, 1993, 3: 81-86. [Medline] [CrossRef]

6) Ozgocmen S, Kaya H, Fadillioglu E, et al.: Effects of calcitonin, risedronate, and raloxifene on erythrocyte antioxidant enzyme activity, lipid peroxidation, and nitric oxide in postmenopausal osteoporosis. Arch Med Res, 2007, 38: 196-205. [Medline] [CrossRef]

7) Merly SL: Metabolic bone diseases. In: Textbook of Rheumatology. Philadelphia: WB Saunders Company, 1997, pp 1563-1581.

8) Ershler WB, Harman SM, Keller ET: Immunologic aspects of osteoporosis. Dev Comp Immunol, 1997, 21: 487-499. [Medline] [CrossRef]

9) Arnett TR, Gibbons DC, Utting JC, et al.: Hypoxia is a major stimulator of osteoclast formation and bone resorption. J Cell Physiol, 2003, 196: 2-8. [Medline] [CrossRef]

10) Vural P, Akgül C, Canbaz M: Effects of menopause and tibolone on antioxidants in postmenopausal women. Ann Clin Biochem, 2005, 42: 220-223. [Medline] [CrossRef]

11) Goktaş UB, Bilgihan A, Ozel U, et al.: Oxidatıve stress in postmenopausal status; Aopp (Advanced Oxidation Proten Products) and lipid peroxidation. Turk J Biochem, 2005, 30: 100

12) Erel O: A new automated colorimetric method for measuring total oxidant status. Clin Biochem, 2005, 38: 1103-1111. [Medline] [CrossRef]

13) Erel O: A novel automated direct measurement method for total antioxidant capacity using a new generation, more stable ABTS radical cation. Clin Biochem, 2004, 37: 277-285. [Medline] [CrossRef]

14) Ulas T, Buyukhatipoglu H, Kirhan I, et al.: Evaluation of oxidative stress parameters and metabolic activities of nurses working day and night shifts. Rev Esc Enferm USP, 2013, 47: 471-476. [Medline]

15) Ulas T, Buyukhatipoglu H, Kirhan I, et al.: The effect of day and night shifts on oxidative stress and anxiety symptoms of the nurses. Eur Rev Med Pharmacol Sci, 2012, 16: 594-599. [Medline]

16) Assessment of fracture risk and its application to screening for postmenopausal osteoporosis. Report of a WHO Study Group. World Health Organ Tech Rep Ser, 1994, 843: 1-129. [Medline]

17) Lippuner $K$, von Overbeck J, Perrelet R, et al.: Incidence and direct medical costs of hospitalizations due to osteoporotic fractures in Switzerland. Osteoporos Int, 1997, 7: 414-425. [Medline] [CrossRef]

18) Cavdar C, Sifil A, Camsarı T: Reactive oxygen particles and antioxidants in the pathogenesis and treatment of diseases. Turkish Nephrology. Dial Transplant J, 1997, 3-4: 96-101.

19) Zaidi M, Alam AS, Bax BE, et al.: Role of the endothelial cell in osteoclast control: new perspectives. Bone, 1993, 14: 97-102. [Medline] [CrossRef]

20) Wimalawansa SJ, De Marco G, Gangula $P$, et al.: Nitric oxide donor alleviates ovariectomy-induced bone loss. Bone, 1996, 18: 301-304. [Medline] [CrossRef]

21) Lee NK, Choi YG, Baik JY, et al.: A crucial role for reactive oxygen species in RANKL-induced osteoclast differentiation. Blood, 2005, 106 852-859. [Medline] [CrossRef]

22) Lean JM, Davies JT, Fuller K, et al: A crucial role for thiol antioxidant in estrogen-deficiency bone loss. J Clin Invest, 2003, 112: 915-923. [Medline] [CrossRef]

23) Maggio D, Barabani M, Pierandrei M, et al.: Marked decrease in plasma antioxidants in aged osteoporotic women: results of a cross-sectional study. J Clin Endocrinol Metab, 2003, 88: 1523-1527. [Medline] [CrossRef]

24) Ratnam DV, Ankola DD, Bhardwaj V, et al.: Role of antioxidants in prophylaxis and therapy: a pharmaceutical perspective. J Control Release, 2006, 113: 189-207. [Medline] [CrossRef]

25) Sontakke AN, Tare RS: A duality in the roles of reactive oxygen species with respect to bone metabolism. Clin Chim Acta, 2002, 318: 145-148. [Medline] [CrossRef]

26) Altindag O, Erel O, Soran N, et al.: Total oxidative/anti-oxidative status and relation to bone mineral density in osteoporosis. Rheumatol Int, 2008 , 28: 317-321. [Medline] [CrossRef]

27) Wolf RL, Cauley JA, Pettinger M, et al.: Lack of a relation between vita- 
min and mineral antioxidants and bone mineral density: results from the Women's Health Initiative. Am J Clin Nutr, 2005, 82: 581-588. [Medline]

28) Swaminathan R: Biochemical markers of bone turnover. Clin Chim Acta 2001, 313: 95-105. [Medline] [CrossRef]

29) Garnero P, Sornay-Rendu E, Chapuy MC, et al.: Increased bone turnover in late postmenopausal women is a major determinant of osteoporosis. J Bone Miner Res, 1996, 11: 337-349. [Medline] [CrossRef]

30) Ohta $H$, Ikeda $T$, Masuzawa $T$, et al.: Differences in axial bone minera density, serum levels of sex steroids, and bone metabolism between postmenopausal and age- and body size-matched premenopausal subjects.
Bone, 1993, 14: 111-116. [Medline] [CrossRef]

31) Miura H, Yamamoto I, Yuu I, et al.: Estimation of bone mineral density and bone loss by means of bone metabolic markers in postmenopausal women. Endocr J, 1995, 42: 797-802. [Medline] [CrossRef]

32) Senocak O, Peker O, Akalın E, et al.: [Bone biochemical marker levels in postmenopausal women with and without osteoporosis]. Turk Fiz Tip Rehab Derg 1999, 2: 41-44.

33) Vs K, K P, Ramesh M, et al.: The association of serum osteocalcin with the bone mineral density in post menopausal women. J Clin Diagn Res, 2013, 7: 814-816. [Medline] 